Communications in Physics, Vol. 29, No. 3 (2019), pp. 241-250

DOI:10.15625/0868-3166/29/3/13756

\title{
TUNABLE CLOAKING OF MEXICAN-HAT CONFINED STATES IN BILAYER SILICENE
}

\author{
LE BIN HO ${ }^{1,2}$ AND LAN NGUYEN TRAN $2, \dagger$ \\ ${ }^{1}$ Department of Physics, Kindai University, Higashi-Osaka 577-8502, Japan \\ ${ }^{2}$ Ho Chi Minh City Institute of Physics, VAST, Ho Chi Minh City, Vietnam \\ ${ }^{\dagger}$ E-mail: lantrann@gmail.com
}

Received 16 April 2019

Accepted for publication 9 July 2019

Published 1 August 2019

\begin{abstract}
We present the ballistic quantum transport of a p-n-p bilayer silicene junction in the presence of spin-orbit coupling and electric field using a four-band model. The transfer-matrix approach has been implemented to evaluate the electron transmission. A Mexican-hat shape of low-energy spectrum is observed similarly to biased bilayer graphene. We show that while bilayer silicene shares some physics with bilayer graphene, it has many intriguing phenomena that have not been reported for the latter. First, there is a significantly non-zero transmission in the Mexican hat, implying the existence of a confined state within the Mexican hat. Second, when the incident energy is below the potential height, the transmission cloaking of this confined state results in a strong oscillation of conductance. Finally, when the incident energy is above the potential height, unlike monolayer silicene the conductance increases with the rise of electric field.
\end{abstract}

Keywords: bilayer silicene; transmission cloaking; transfer-matrix method.

Classification numbers: 61.46.-w; 61.48.Gh; 81.07.-b.

(C)2019 Vietnam Academy of Science and Technology 


\section{INTRODUCTION}

Unlike monolayer graphene, bilayer graphene has a parabolic dispersion relation and no Klein tunneling is observed [1,2]. In a certain region of incident energy, the chirality mismatch of states inside and outside a $p-n-p$ junction leads to a cloaking of transmission $[3,4]$. More interestingly, applying different electrostatic potentials at the two layers of bilayer graphene, called biased bilayer graphene, results in a tunable band gap and Mexican-hat shape of low-energy spectrum [5]. Great efforts both in theory and experiment have been devoted to reproduce and explain these phenomena [5-7]. Thanks to its peculiar electronic structures, biased bilayer graphene was proposed as a new platform for electronic devices, such as the low-voltage tunnel switches [8]. Moreover, some recent studies have revealed a hydrogen-like bound state within Mexican hat opening a new door for biased bilayer graphene applications [9].

While sharing some intriguing properties of graphene, silicene, a two-dimensional allotrope of silicon, has some superior advantages compared to graphene, such as strong spin-orbit coupling (SOC) and buckled honeycomb structure. While SOC enables us to realize the quantum spin Hall effect [10], the buckled honeycomb structure help us control the bulk band gap of silicene by applying an external electric field [11]. Topological phase transitions and quantum transport properties of monolayer silicene in the presence of external fields, such as electric and exchange fields, and circularly polarized light in the off-resonant regime, have been extensively reported [12-14].

Apart from monolayer, bilayer silicene were also successfully synthesized in experiment. It is expected that bilayer silicene can provide some unusual physics that cannot be found in monolayer. Recently, there have been many theoretical works focusing on the topological phase transitions, magneto-optical, and optoelectronic properties of bilayer silicene, for instances, see Refs. [15-17]. Nevertheless, its quantum transport properties still remain unexplored. As seen from bilayer graphene, the two-band model is insufficient in the presence of a strong interlayer bias even at the Dirac point $[4,5]$. Therefore, the four-band model is essential in order to properly describe the low-energy physics of bilayer silicene.

In this paper, we investigate ballistic transport properties of a $p-n-p$ bilayer silicene junction in the presence of a transverse electric field using the four-band low-energy model. The transfermatrix approach was implemented to evaluate the electron transmission. Some novel quantumtransport properties of bilayer silicene that have not been reported for monolayer silicene and bilayer graphene will be discussed.

\section{THEORY}

\section{II.1. Model and electronic structure}

While there are four possibilities of $A B$ bilayer stacking [15], we only consider the forward stacking configuration displayed in Fig. 1 and the same investigation can be done for the other configurations. As seen in the figure, bilayer silicene are composed of two silicene monolayers having an in-plane interatomic distance $a=2.46 \AA$. Each layer has a buckled structure consisting of two nonequivalent sublattices denoted by $A$ and $B$. The intralayer atomic distance is $2 l$ with $l=0.23 \AA$. The spin-orbit coupling $\lambda_{S O}$ and the intralayer coupling between $A$ and $B$ atoms $t_{0}$ are $3.9 \mathrm{meV}$ and $1.6 \mathrm{eV}$, respectively. The two layers are stacked according to the $A_{2} B_{1}$ stacking, e.g. $B_{1}$ right above $A_{2}$, with a distance $2 L$. In this work, $L$ is fixed at $1.46 \AA$. As shown in Fig. 1 , the 
perpendicular interlayer coupling between the $A_{2}$ and $B_{1}$ atoms is $t_{A_{2} B_{1}}=t_{\perp}$, while those between the other interlayer atom pairs are $t_{A_{1} B_{2}}=t_{3}$ and $t_{A_{1} A_{2}}=t_{B_{1} B_{2}}=t_{4}$. The interlayer skew hopping term $t_{3}$ results in a so-called trigonal warping occurring only at very low energies. The second skew hopping term, $t_{4}$, has a tiny impact on the electronic properties. Therefore, we have not included these two hopping terms in the current work.

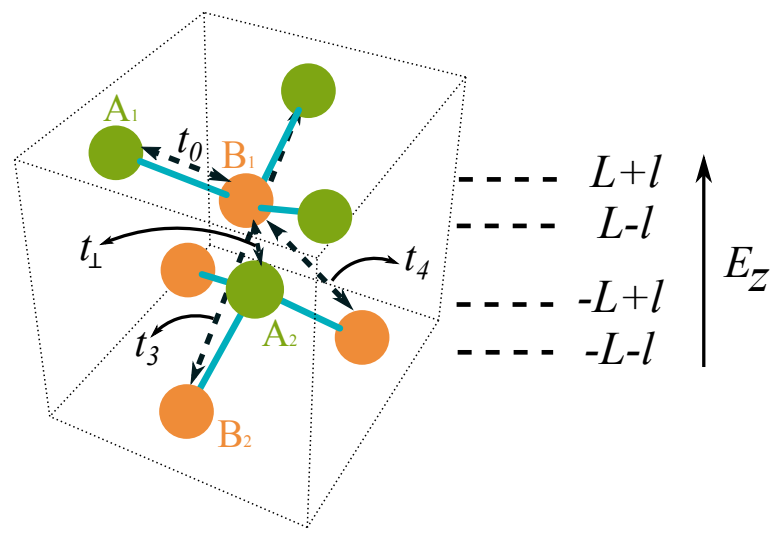

Fig. 1. The unit cell of bilayer silicene with the forward $A B$ stacking configuration. Green and orange indicate the two sublattices A and B of monolayer, respectively. The interlayer and intralayer sublattice distances are $2 L$ and $2 l$, respectively. While $t_{0}$ is the intralayer hoping, $t_{\perp}$ is the perpendicular interlayer hoping. In the current work, two interlayer skew hoping $t_{3}$ and $t_{4}$ are not included.

Following the continuum nearest-neighbor tight-binding formalism, the effective Hamiltonian near the Dirac points and the eigenstate are given by [15]

$$
H=\left(\begin{array}{cccc}
U+m_{+} & v_{F} \pi & t_{\perp} & 0 \\
v_{F} \pi^{\dagger} & U+m_{-} & 0 & 0 \\
t_{\perp} & 0 & U-m_{+} & v_{F} \pi^{\dagger} \\
0 & 0 & v_{F} \pi & U-m_{-}
\end{array}\right), \Psi=\left(\begin{array}{l}
\psi_{A_{1}} \\
\psi_{B_{1}} \\
\psi_{B_{2}} \\
\psi_{A_{2}}
\end{array}\right),
$$

where $v_{F} \approx 5.5 \times 10^{5} \mathrm{~m} / \mathrm{s}$ is the Fermi velocity of the charge carries in silicene, $\pi=p_{x}+i p_{y}$ and $\boldsymbol{p}$ is the momentum operator, $U$ is an external potential. The terms $m_{ \pm}$represent the contribution of SOC $\left(\lambda_{S O}\right)$ and electric field $E_{z}$. For the forward stacking configuration considered here, we have $m_{ \pm}=\mp \lambda_{S O}+(L \pm l) E_{z}$. Using dimensionless variables: $\epsilon=(E-U) / t_{\perp}$ and $k_{y} \rightarrow \hbar v_{F} k_{y} / t_{\perp}$, we can write the eigenvalues $E$ of the Hamiltonian $H$ as follows,

$$
\epsilon=\eta \frac{1}{\sqrt{2}} \sqrt{\beta+\theta \sqrt{\beta^{2}-4 \alpha}}
$$

with

$$
\begin{aligned}
& \beta=1+m_{+}^{2}+m_{-}^{2}+2 k^{2}, \\
& \alpha=\left(k^{2}-m_{+} m_{-}\right)^{2}+m_{-}^{2}, \\
& k=\sqrt{k_{x}^{2}+k_{y}^{2}} .
\end{aligned}
$$


In Eq. (2), while the index $\eta= \pm 1$ corresponds to conducting ( + ) and valence (-) bands, the index $\theta= \pm 1$ represents the low-energy (-) and high-energy (+) branches. As seen in the left panel of Fig. 2, the low-energy branches $(\theta=-1)$ of band structure (2) displays an unique Mexican-hat shape.
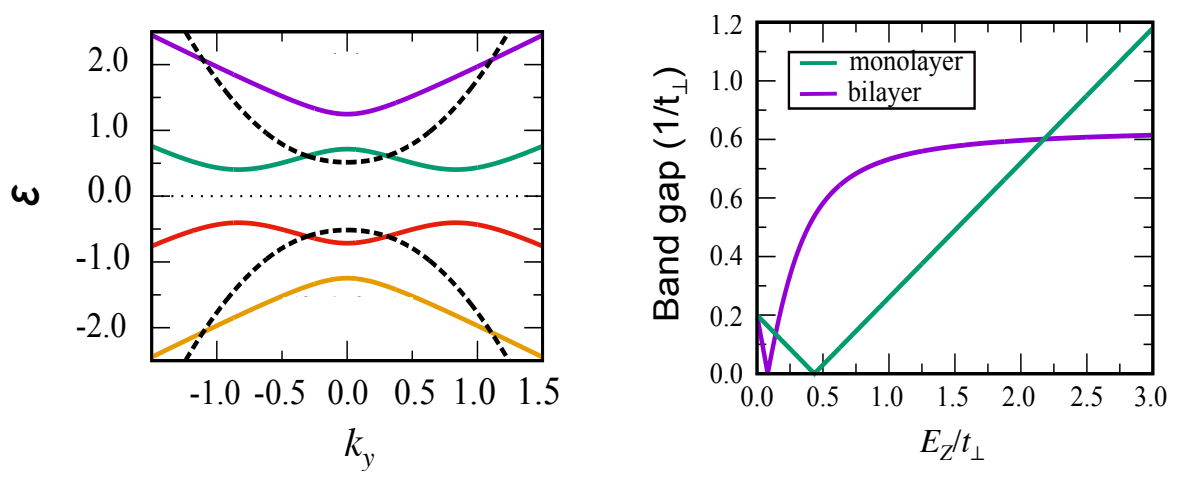

Fig. 2. Left panel: band structures of bilayer silicene with $\lambda_{S O}=0.1 t_{\perp}, E_{z}=0.5 t_{\perp}$, and $U=0$. The dashed black curves are the two-band spectrum. Right panel: the band gap of bilayer silicene as functions of electric field $E_{z}$. For comparison, the monolayer result is also plotted.

The right panel of Fig. 2 represents the variation of bilayer silicene band gap with the electric field $E_{z}$. For comparison, the monolayer result is also provided. Critical points where the band gaps are closed are observed for both systems. However, it is lower for the bilayer than for the monolayer. For $E_{z}>t_{\perp}$, while the monolayer band gap linearly increases beyond the critical point, the bilayer one is almost unchanged.

\section{II.2. Ballistic transport}

We now model a one-dimensional square well potential $U(x)$ of a width $d$ applied equally to the two layers of bilayer silicene as follows

$$
U(x)= \begin{cases}U & \text { if } 0 \leq x \leq d(\text { region } 2) \\ 0 & \text { if } x<0 \text { or } x>d(\text { region } 1 \text { or } 3) .\end{cases}
$$

Similarly, the electric field is only applied to the region 2. With the translational invariance along the $y$ direction, i.e. the momentum $k_{y}$ is unchanged during electron motion, the wave function can be written as $\Psi(x, y)=\psi(x) e^{i k_{y} y}$. Solving the time-independent Schrodinger equation $H \Psi=E \Psi$ we obtain the eigenstates, that are given as

$$
\psi(x)=\left(\begin{array}{l}
\psi_{A_{1}} \\
\psi_{B_{1}} \\
\psi_{B_{2}} \\
\psi_{A_{2}}
\end{array}\right)=\mathscr{P} \mathscr{Q}(x) C,
$$


Here, $C$ are wavefunction coefficients, $\mathscr{Q}(x)=\operatorname{diag}\left(e^{i k_{+} x}, e^{-i k_{+} x}, e^{i k_{-} x}, e^{-i k_{-} x}\right)$ and

$$
\mathscr{P}=\left(\begin{array}{cccc}
1 & 1 & 1 & 1 \\
f_{+}^{+} & f_{-}^{+} & f_{+}^{-} & f_{-}^{-} \\
g^{+} & g^{+} & g^{-} & g^{-} \\
h_{+}^{+} & h_{-}^{+} & h_{+}^{-} & h_{-}^{-}
\end{array}\right) .
$$

with,

$$
\begin{aligned}
f_{ \pm}^{\eta} & =\left( \pm k_{\eta}-i k_{y}\right) /\left(\epsilon-m_{-}\right), \\
g^{\eta} & =\left[-k_{\eta}^{2}-k_{y}^{2}+\gamma_{-}\right] /\left(\epsilon-m_{-}\right), \\
h_{ \pm}^{\eta} & =\left[-k_{\eta}^{2}-k_{y}^{2}+\gamma_{-}\right]\left( \pm k_{\eta}+i k_{y}\right) /\left(\epsilon^{2}-m_{-}^{2}\right),
\end{aligned}
$$

where

$$
k_{\eta}=\sqrt{\frac{\gamma_{+}+\gamma_{-}}{2}+\eta \sqrt{\Delta}-k_{y}^{2}}
$$

and

$$
\begin{aligned}
\gamma_{ \pm} & =\left(\epsilon \pm m_{-}\right)\left(\epsilon \pm m_{+}\right), \\
\Delta & =\frac{\left(\gamma_{+}+\gamma_{-}\right)^{2}}{4}+\left(\epsilon^{2}-m_{-}^{2}\right) .
\end{aligned}
$$

$k_{\eta}$ is the wave vector in the $x$ direction, with $\eta= \pm 1$. It is derived from the dispersion relation (Eq. (2)). The index $\eta$ now corresponds to the pseudospin state of particles. Whenever $\epsilon \geq \lambda_{S O}$, which is the case we consider in this paper, the wave vector $k_{+}$is always real. The wave vector $k_{-}$, however, can be either real or imaginary due to the relation of the value $\epsilon$ to $\lambda_{S O}$, and $k_{y}$. For the normal incident $\left(k_{y}=0\right)$, when $\lambda_{S O}<\epsilon<\sqrt{1+\lambda_{S O}^{2}}, k_{-}$is imaginary. Therefore, the propagation only happens for the $k_{+}$mode. When $\epsilon>\sqrt{1+\lambda_{S O}^{2}}, k_{-}$becomes real. As a result, the propagation is carried out by both modes. Corresponding to these two distinct propagate modes, there are two non-scattering transmission channels as $T_{+}^{+}$and $T_{-}^{-}$for propagation via $k_{+}$and $k_{-}$modes, respectively. There also exists two others scattering channels: $T_{-}^{+}$for scattering from $k_{+}$to $k_{-}$and $T_{+}^{-}$for scattering from $k_{-}$to $k_{+}$.

In the limit $\epsilon \ll t_{\perp}$ and with an assumption that $m_{ \pm}$and $\epsilon$ are the same order of magnitude, by neglecting the second order of $\epsilon$ and $m_{ \pm}$in Eq. (6), the two-band model can be obtained $[4,5]$. As displayed in the left panel of Fig. 2, the two-band model (the dashed black curves) is unable to yield the Mexican-hat shape. We therefore will not discuss it further in this paper.

The continuity of wave functions at $x=0$ and $x=d$ gives the boundary conditions $\psi_{1}(0)=$ $\psi_{2}(0)$ and $\psi_{2}(d)=\psi_{3}(d)$. The transfer matrix $\mathscr{M}$ can be then written as

$$
\mathscr{M}=\mathscr{P}_{1}^{-1} \mathscr{P}_{2} \mathscr{Q}_{2}^{-1}(d) \mathscr{P}_{2}^{-1} \mathscr{P}_{3} \mathscr{Q}_{3}(d),
$$

and the components of the vector $C$ in the region I and III are given:

$$
C_{I}^{\eta}=\left(\begin{array}{c}
\delta_{\eta, 1} \\
r_{+}^{\eta} \\
\delta_{\eta,-1}^{\eta} \\
r_{-}^{\eta}
\end{array}\right) \text {, and } C_{I I I}^{\eta}=\left(\begin{array}{c}
t_{+}^{\eta} \\
0 \\
r_{-}^{\eta} \\
0
\end{array}\right)
$$


with $\eta= \pm 1$. By taking into account the change in velocity of the waves scattering into different modes, the transmissions $T$ are given by

$$
T_{ \pm}^{\eta}=\frac{k_{ \pm}}{k_{\eta}}\left|t_{ \pm}^{\eta}\right|^{2}
$$

Finally, according to Landauer-Büttiker formalism, the normalized spin-valley dependent conductance at zero temperature is evaluated as

$$
G=\frac{1}{2} \int_{-\pi / 2}^{\pi / 2} \sum T_{ \pm}^{ \pm}(E, \phi) \cos (\phi) d \phi
$$

where $\phi$ is the incident angle.

\section{NUMERICAL RESULTS}

In unbiased bilayer graphene, the cloaking effect of transmission through a barrier was observed at the normal incidence [3,4]. This can be briefly explained as follows. Let us consider a propagation via the $k_{+}$mode as displayed in Fig. 3. For the normal incidence $\left(k_{y}=0\right)$, the pseudospin is conserved. This means that the $k_{+}$mode outside the barrier can only couple with the $k_{+}$mode inside the barrier. However, the energy spectrum inside the barrier is shifted, leading to the mismatch between $k_{+}$modes inside and outside the barrier. Even though there are $k_{-}$ states available inside the barrier, the propagation via the $k_{+}$mode through the barrier is unlikely, resulting in the transmission cloaking inside the barrier.

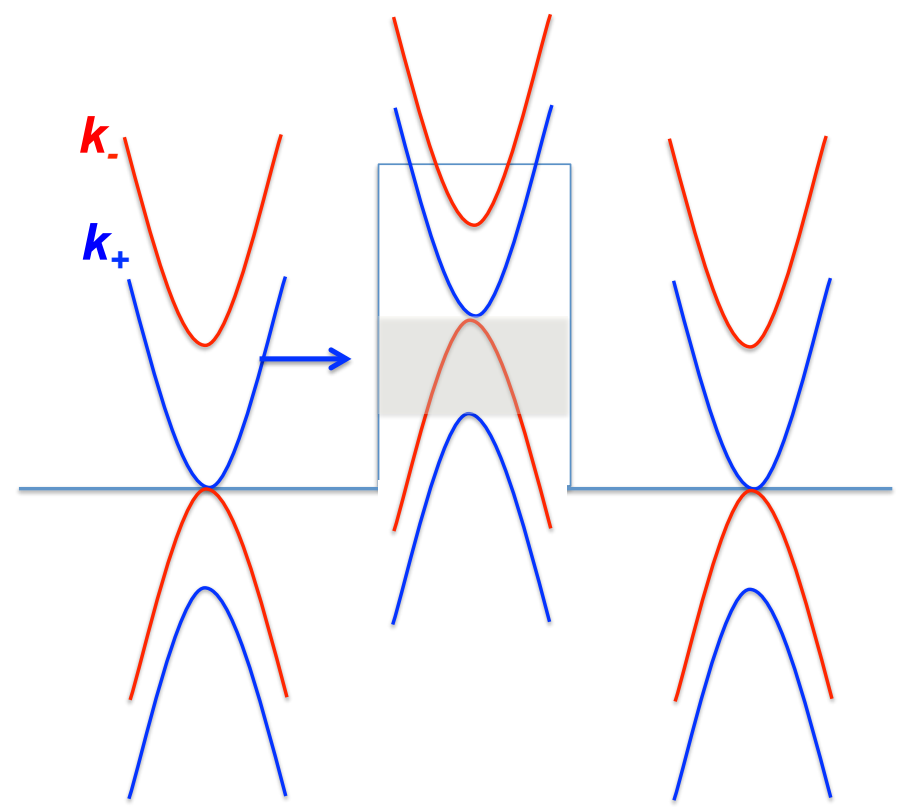

Fig. 3. Schematic representation of energy spectra of unbiased bilayer graphene inside and outside the potential barrier. The arrow indicates the direction of propagation. The transmission cloaking of $k_{+}$mode occurs in the gray region where there are no available $k_{+}$states inside the barrier. 


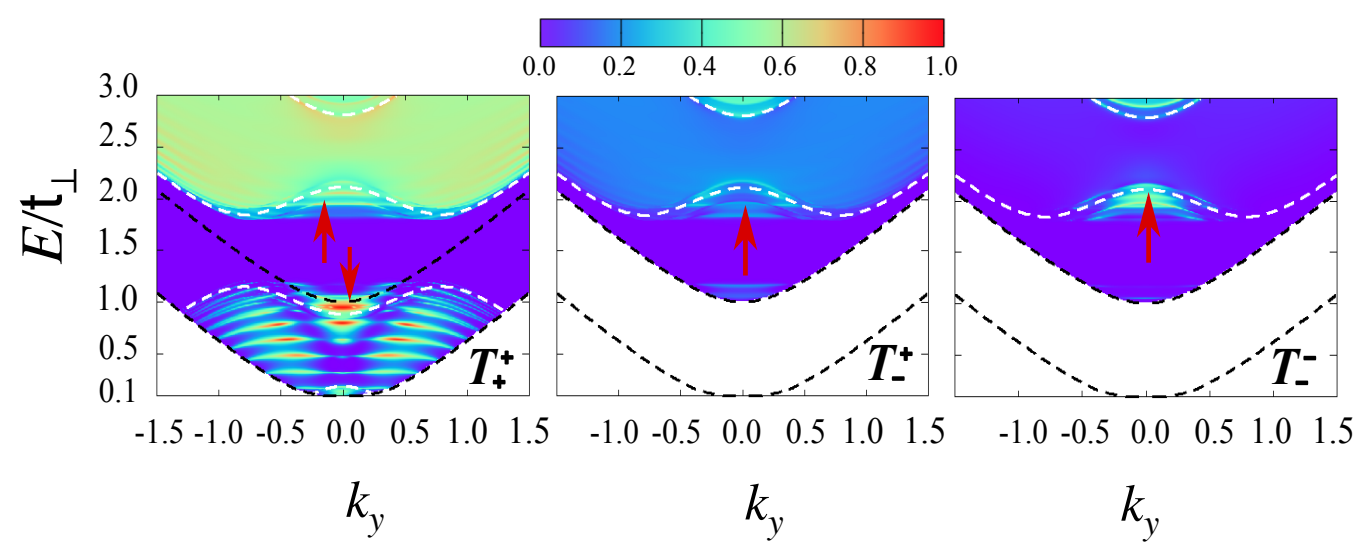

Fig. 4. Transmission spectra of different modes $\left(T_{+}^{+}, T_{-}^{+}=T_{+}^{-}\right.$, and $\left.T_{-}^{-}\right)$as functions of incident energy and transverse wave vector $k_{y}$ in the presence of SOC $\lambda_{S O}=0.1 t_{\perp}$ and electric field $E_{z}=0.5 t_{\perp}$. The white dashed curves are the four-band dispersion spectra, whereas the black dashed curves are the border between the propagating and evanescent regions. The red arrows indicate the non-zero transmission within the Mexican hat. The height of potential barrier is $1.5 t_{\perp}$.

The transmission spectra of bilayer silicene in the presence of SOC and electric field are displayed in Fig. 4. Since the electric field $E_{z}$ modifies the particles' momenta $k_{\eta}$ inside the barrier (Eq. (6)), the cloaking in the $T_{+}^{+}$channel splits into two bpranches at finite $k_{y}$. The splitting of the transmission cloaking was also found for bilayer graphene in the presence of interlayer bias [4]. One fascinating feature that was not reported for bilayer graphene is that transmission within the Mexican hats is significantly non-zero for all channels indicated by red arrows in the figure, implying the existence of confined states in these regions, called the Mexican-hat confined states. We would like to emphasize that one should not be confused with states confined in a potential barrier, the Mexican-hat confined state is formed in the Mexcian-hat region of band structures under an external electric field.

Let us now investigate the conductance of bilayer silicene. As seen in Fig. 2, there is a linear dependence of monolayer band gap on electric field, resulting in a monotonic decrease of monolayer conductance. Based on what we have observed for bilayer silicene, it is expected that new phenomena can be observed. Fig. 5a represents the conductance as a function of incident energy $E$ and electric field $E_{z}$ when $E<U$. Interestingly, unlike monolayer, the bilayer conductance strongly oscillates with respect to $E_{z}$. As seen in Fig. 4, for $E<U$ the conductance is dominated by the channel $T_{+}^{+}$. Therefore, in order to get an insight into the oscillation of the conductance, we plot in Fig. 6 the $T_{+}^{+}\left(E, k_{y}\right)$ spectrum at three selected $E_{z}$ values corresponding to two peaks $\left(0.9 t_{\perp}\right.$ and $\left.1.05 t_{\perp}\right)$ and one valley $\left(0.98 t_{\perp}\right)$ of the conductance. Clearly, the $T_{+}^{+}$transmission of valence band is mainly contributed by the confined state in the Mexican hat. Strong Fabry-Perot resonances of transmission spectra imply the discretization of these states. Furthermore, the transmission within the Mexican hat also oscillates with respect to the wave vector $k_{y}$. At $E_{z}=0.9 t_{\perp}$, the large cloaking region around the normal incidence $\left(k_{y}=0\right)$ significantly suppresses the conductance. On the other hand, at $E_{z}=0.98 t_{\perp}$, the cloaking shifts to finite $k_{y}$ and is not significant, 


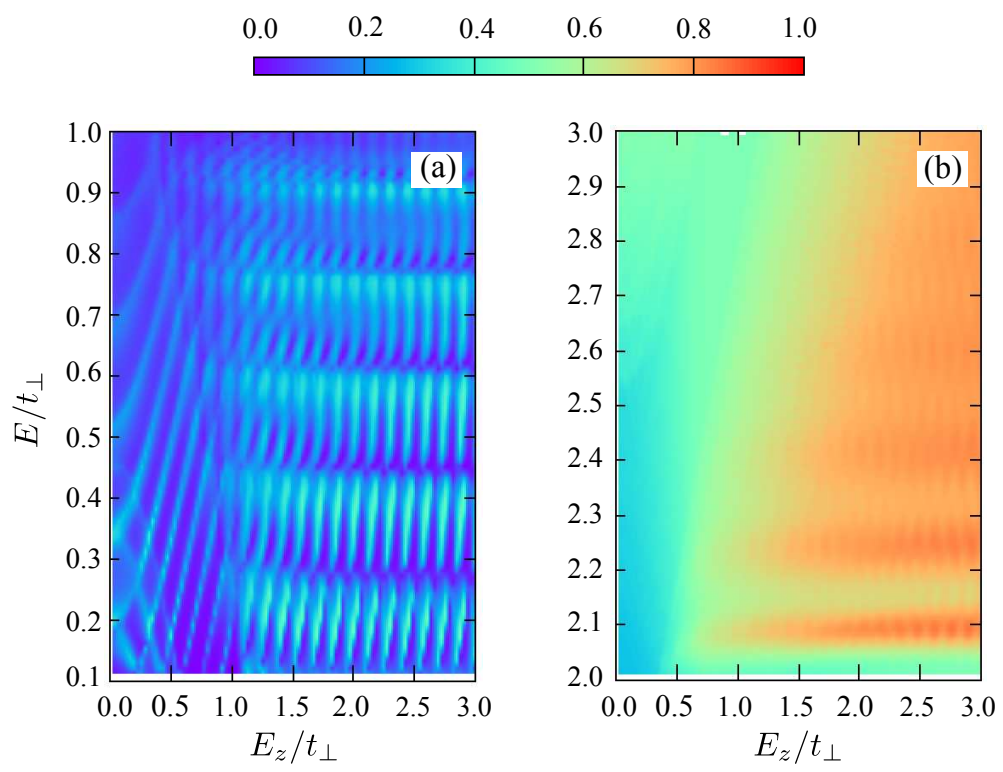

Fig. 5. Conductance as a function of incident energy $E$ and electric field $E_{z}$ for $E$ below (a) and above (b) the potential height that is set at $U=1.5 t_{\perp}$.

leading to an enhancement of conductance. Finally, at $E_{z}=1.05 t_{\perp}$, the conductance is again lowered due to a large cloaking at the normal incidence. In general, one can conclude that the cloaking at the normal incidence within the Mexican hat causes the oscillation of conductance.

What is the origin of the transmission cloaking in the Mexican hat? It is believed not due to a shift of energy spectrum as in the case of barrier potential discussed above. Recently, Skinner and coworkers [9] have found a hydrogen-like bound state within Mexican hat of biased bilayer graphene. They showed that the bound state's electron density strongly oscillates with respect to the wave vector $k$ as the applied bias increases. Following this argument, we may attribute the cloaking of the confined state inside Mexican hat to the oscillation of its electron density. For example, at $E_{z}=0.9 t_{\perp}$, the confined state's electron density is almost zero around the normal incidence. Therefore, it does not show up in the normal incidence transmission. In contrast, the confined state's electron density around the normal incidence is largely non-zero at $E_{z}=0.98 t_{\perp}$. As a result, the normal incidence propagation via Mexican hat is allowed. In order to more convincingly demonstrate the cloaking of Mexican-hat confined state, an analytic relation between its electron density and electric field is essentially derived. However, it is beyond the scope of the current work and we would leave it for a future work.

Figure 5 represents the conductance as a function of incident energy $E$ and electric field $E_{z}$ when $E>U$. Even though there is also a large transmission cloaking in the Mexican hat, the oscillation of conductance is less significant than it is when $E<U$. As seen in Fig. 6, the transmission for the conducting band is contributed from both states inside and outside the Mexican hat. On the other hand, the band gap tends to a saturation when $E_{z}>1.0 t_{\perp}$ as seen in Fig. 2. Consequently, unlike monolayer, enlarging the interlayer distance results in an increasing of conductance with the electric field $E_{z}$. 


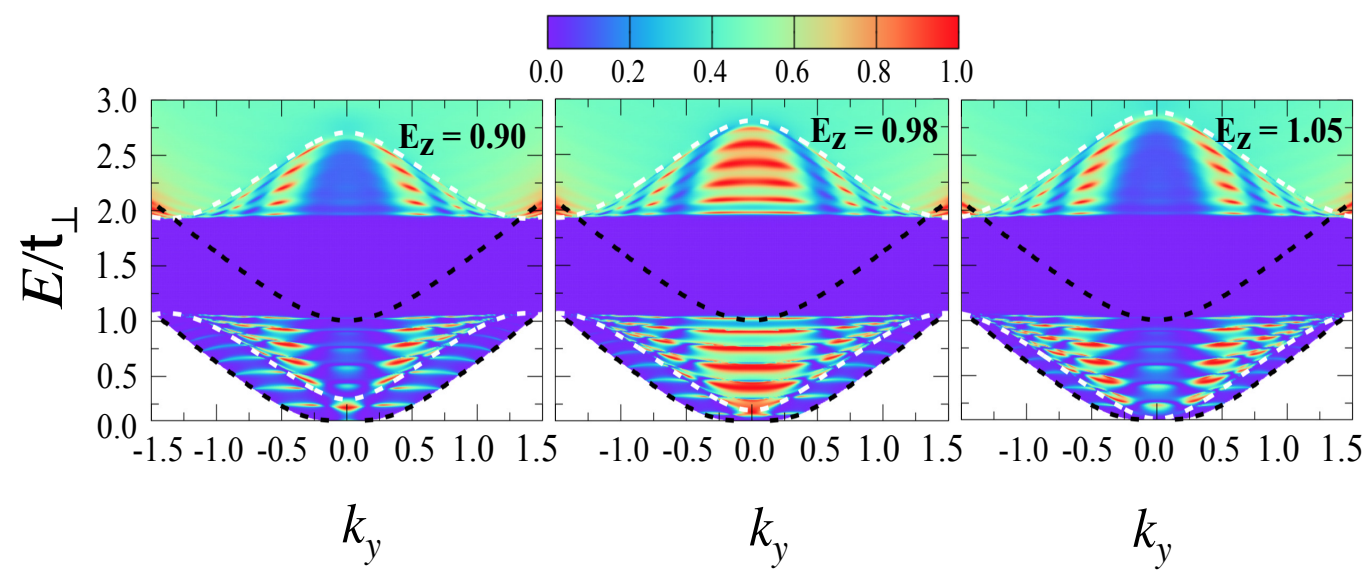

Fig. 6. $T_{+}^{+}$as a function of incident energy $E$ and transverse wave vector $k_{y}$ at different value of $E_{z}$. The white dashed curves are the four-band dispersion spectra, whereas the black dashed curves are the border between the propagating or evanescent regions. The potential height $U=1.5 t_{\perp}$.

\section{CONCLUSIONS}

In conclusions, we have presented the ballistic transport of a $p$ - $n$ - $p$ bilayer silicene junction in the presence of both SOC and electric field using the four-band model and the transfer-matrix approach. We observed the Mexican-hat shape of low-energy spectra similarly to biased bilayer graphene. We found that the confined state produces the non-zero transmission within the Mexican hat. Furthermore, the cloaking of this confined state results in a strong oscillation of conductance with respect to electric field when the incident energy is below the potential height. On the other hand, unlike monolayer, the conductance of bilayer silicene is slowly enhanced under electric field when the incident energy is above the potential height. Our theoretical results are believed to be useful for realistic applications of bilayer silicene in electronics, such as field effect transistors or electronic switches. Working on an analytic relation between the Mexican-hat confined state's electron density and electric field is on progress.

\section{ACKNOWLEDGMENT}

This work was supported by Vietnamese National Foundation of Science and Technology Development (NAFOSTED) under Grant No. 103.01-2015.14.

\section{REFERENCES}

[1] E. McCann and M. Koshino, Rep. Prog. Phys. 76 (2013) 056503.

[2] M. Katsnelson, K. Novoselov and A. Geim, Nat. Phys. 2 (2006) 620.

[3] N. Gu, M. Rudner and L. Levitov, Phys. Rev. Lett. 107 (2011) 156603.

[4] B. Van Duppen and F. Peeters, Phys. Rev. B 87 (2013) 205427.

[5] E. V. Castro, K. S. Novoselov, S. V. Morozov, N. Peres, J. L. Dos Santos, J. Nilsson, F. Guinea, A. Geim and A. C. Neto, J. Phys.: Condens. Matter 22 (2010) 175503.

[6] K. Lee, S. Lee, Y. S. Eo, C. Kurdak and Z. Zhong, Phys. Rev. B 94 (2016) 205418. 
[7] K. F. Mak, C. H. Lui, J. Shan and T. F. Heinz, Phys. Rev. Lett. 102 (2009) 256405.

[8] G. Alymov, V. Vyurkov, V. Ryzhii and D. Svintsov, Sci. Rep. 6 (2016) 24654.

[9] B. Skinner, B. Shklovskii and M. Voloshin, Phys. Rev. B 89 (2014) 041405.

[10] C.-C. Liu, W. Feng and Y. Yao, Phys. Rev. Lett. 107 (2011) 076802.

[11] N. D. Drummond, V. Zólyomi and V. I. Fal'ko, Phys. Rev. B 85 (2012) 075423.

[12] M. Ezawa, Phys. Rev. Lett. 109 (2012) 055502.

[13] M. Ezawa, Phys. Rev. Lett. 110 (2013) 026603.

[14] L. B. Ho and T. N. Lan, J. Phys. D: Appl. Phys. 49 (2016) 375106.

[15] M. Ezawa, J. Phys. Soc. Japan 81 (2012) 104713.

[16] H. Da, W. Ding and X. Yan, Appl. Phys. Lett. 110 (2017) 141105.

[17] B. Huang, H.-X. Deng, H. Lee, M. Yoon, B. G. Sumpter, F. Liu, S. C. Smith and S.-H. Wei, Phys. Rev. X 4 (2014) 021029. 